\title{
Magnetocrystalline Anisotropy in a Single Crystal Fe-Ni-Ga Ferromagnetic Shape Memory Alloy*1
}

\author{
Haruhiko Morito ${ }^{1, * 2}$, Asaya Fujita ${ }^{1}$, Kazuaki Fukamichi ${ }^{1}$, Takuya Ota ${ }^{1, * 2}$, Ryosuke Kainuma ${ }^{1}$, \\ Kiyohito Ishida ${ }^{1}$ and Katunari Oikawa ${ }^{2}$ \\ ${ }^{1}$ Department of Materials Science, Graduate School of Engineering, Tohoku University, Sendai 980-8579, Japan \\ ${ }^{2}$ National Institute of Advanced Industrial Science and Technology, Tohoku Center, Sendai 983-8551, Japan
}

\begin{abstract}
In order to discuss the advent of the magnetic-field-induced strains for a single crystal $\mathrm{Fe}_{22 .} \mathrm{Ni}_{51.5} \mathrm{Ga}_{26.5}$ shape memory alloy, the magnetic easy axis in the $14 \mathrm{M}$ martensite phase and the magnetocrystalline anisotropy constant $K$ have been investigated. From the data on the spontaneous thermal expansion and the magnetization curves, the magnetic easy axis of the $14 \mathrm{M}$ martensite phase was determined to be the $[010]_{14 \mathrm{M}}$. The value of $K$ in the martensite phase was estimated to be $1.3 \times 10^{5} \mathrm{~J} / \mathrm{m}^{3}$, implying to exhibit a large value of the magnetic-fieldinduced strain.
\end{abstract}

(Received December 18, 2002; Accepted February 10, 2003)

Keywords: ferromagnetic shape memory alloy, martensitic transformation, Fe-Ni-Ga alloy, magnetocrystalline anisotropy, magnetic-fieldinduced strain

\section{Introduction}

In ferromagnetic shape memory alloys (FSMAs), a thermoelastic martensitic transformation in the ferromagnetic state is controlled by magnetic fields. The existence of the ferromagnetism offers a faster response compared with temperature driven shape memory alloys. In addition, large magnetic-field-induced strains (MFISs) have been observed in the martensite phase in FSMAs, including $\mathrm{Ni}_{2} \mathrm{MnGa},{ }^{1)}$ $\mathrm{FePd},{ }^{2)} \mathrm{FePt}^{3)} \mathrm{Ni}_{2} \mathrm{MnAl}^{4,5)}$ and $\mathrm{CoNiGa}{ }^{6,7)}$ Recently, the present authors have reported $\mathrm{Co}-\mathrm{Ni}-\mathrm{Al}^{7-10}$ alloys as FSMAs. Fast responses and large MFIS are necessary for applications of FSMAs to sensors and actuators.

The MFIS arises from a process of twin-boundary motion in the martensite phase. ${ }^{1,11)}$ When the martensitic transformation takes place, various variants would be formed with keeping coherence with the parent crystal structure during the cooling process. In each variant, the magnetic moment is pinned to the magnetic easy axis due to a large magnetocrystalline anisotropy constant $K$. Furthermore, microscopic magnetic domains exist in martensite variants so as to reduce the magnetic dipole energy. The magnetic domains of which the magnetization $M$ direction is not parallel to the applied magnetic field $H$ direction are diminished on applying $H$, and then $M$ changes its direction to the $H$ direction. When the magnetocrystalline anisotropy energy is larger than the driving energy change to move twin boundaries, the variants change to other variants so that the magnetic easy axis comes close to parallel to the $H$ direction. As mentioned above, the magnitude of the MFIS depends on $K$ and the mobility of twin boundaries. One of the parameters which express the mobility of twin boundaries is the twinning stress. In other words, low twinning stresses are necessary for the twin boundary motions.

Recently, $\mathrm{Fe}-\mathrm{Ni}-\mathrm{Ga}^{12,13)}$ alloys have been drawn attention

\footnotetext{
*1 This Paper was Presented at the Autumn Meeting of the Japan Institute of

Metals, held in Suita, November 4.

${ }^{* 2}$ Graduate Student, Tohoku University.
}

as new FSMAs. These alloys undergo a thermoelastic martensitic transformation. The parent phase has a B2 structure or an L $2_{1}$-Heusler structure by annealing above or below the ordering temperature. After martensitic transformation, the lattice has a 7-layer modulated (14 M) structure and/or a 5-layer modulated (10 M) structure. ${ }^{13)} \mathrm{Ni}_{2} \mathrm{MnGa}$ alloy having the modulated layer structures such as $10 \mathrm{M}$ and $14 \mathrm{M}$ has low twinning stresses, ${ }^{14)}$ and its large magnetocrystalline anisotropy $^{15)}$ is accompanied by a large MFIS. The Fe-Ni-Ga alloy system also has such modulated layer structures in the martensite phase, and hence the twinning stresses to move the twin boundaries are considered to be low. If the $\mathrm{Fe}-\mathrm{Ni}-\mathrm{Ga}$ alloys have a large magnetocrystalline anisotropy, one would expect to observe a large MFIS. Practically, the determination of the easy axis governing the twin boundaries is important. In the present paper, therefore, the magnetocrystalline anisotropy and the easy axis of a single crystal $\mathrm{Fe}-\mathrm{Ni}-\mathrm{Ga} \beta^{\prime}$ martensite phase transformed from the $\beta$ parent phase have been investigated.

\section{Experiment}

A single crystal $\mathrm{Fe}_{22.0} \mathrm{Ni}_{51.5} \mathrm{Ga}_{26.5}$ alloy for the measurement was grown by an optical floating-zone method in a helium atmosphere. The composition of the $\mathrm{Fe}-\mathrm{Ni}-\mathrm{Ga} \beta$ phase was determined by energy dispersion X-ray spectroscopy (EDX). The single crystal was annealed at $1453 \mathrm{~K}$ for $24 \mathrm{~h}$ to homogenize the single phase and followed by quenching in ice water. After homogenization, the specimen was heat-treated at $1023 \mathrm{~K}$ for $1 \mathrm{~h}$, and then slowly furnacecooled to achieve the atomic ordering in the parent phase. The crystallographic orientations in the $\beta$ parent phase were determined from electron backscattering diffraction patterns (EBSPs). The rectangular sample size for the magnetization measurement was $3.0 \mathrm{~mm} \times 3.0 \mathrm{~mm} \times 1.5 \mathrm{~mm}$. The faces of the single crystal were $\{100\}_{\mathrm{P}}$ of the high-temperature parent phase, and the short axis was parallel to the direction $[001]_{P}$. In order to determine the magnetic and martensitic transfor- 
mation temperatures, the thermomagnetization curves were measured in a magnetic field $8 \mathrm{kAm}^{-1}$ on cooling and heating processes. In order to investigate the formation of variants, the spontaneous $(H=0)$ linear thermal expansion (LTE) curve for each direction $[100]_{\mathrm{P}},[010]_{\mathrm{P}}$, and $[001]_{\mathrm{P}}$ in the parent cubic phase was measured in the temperature range between 155 and $300 \mathrm{~K}$. The magnetization curves at $10 \mathrm{~K}$ were obtained with a superconducting quantum interference device (SQUID) magnetometer in the magnetic field up to $0.5 \mathrm{MAm}^{-1}$.

\section{Results and Discussion}

The thermomagnetization curves in a magnetic field of $H=8.0 \mathrm{kAm}^{-1}$ in the cooling and heating processes for the single crystal $\mathrm{Fe}_{22.0} \mathrm{Ni}_{51.5} \mathrm{Ga}_{26.5}$ are shown in Fig. 1. In the cooling process, the increase of magnetization $M$ due to the transition from the paramagnetic to the ferromagnetic state was observed at high temperatures. This transition is of the second-order, and hence the Curie temperature $T_{\mathrm{C}}$ is defined as the minimum point of the derivative of magnetization $d M / d T$. The decrease of $M$ due to the change in the magnetocrystalline anisotropy constant $K$ caused by the martensitic transformation is observed at low temperatures. The change in $M$ between the martensite state and the parent state occurs, because $K$ in the martensite phase is larger than that in the parent phase. In the cooling process, the starting and finishing temperatures which change the value of $M$ are defined as the martensitic transformation starting temperature $M_{\mathrm{s}}$ and its finishing temperature $M_{\mathrm{f}}$, respectively. Since the martensitic transformation is of the first-order, the transformation is accompanied by hysteresis in the thermomagnetization curve. In the heating process, the starting and finishing temperatures that also change $M$ are defined as the reverse transformation starting temperature $A_{\mathrm{s}}$ and the finishing temperature $A_{\mathrm{f}}$, respectively. From the thermomagnetization curves in Fig. 1, the following temperatures are determined

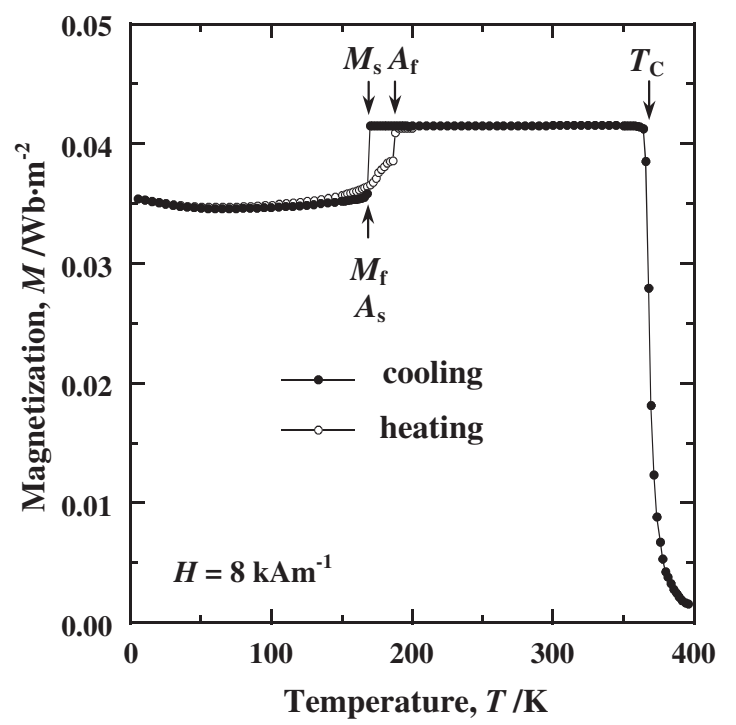

Fig. 1 Thermomagnetization curves in a magnetic field of $8 \mathrm{kAm}^{-1}$ on cooling and heating processes for the single crystal $\mathrm{Fe}_{22.0} \mathrm{Ni}_{51.5} \mathrm{Ga}_{26.5}$ alloy.

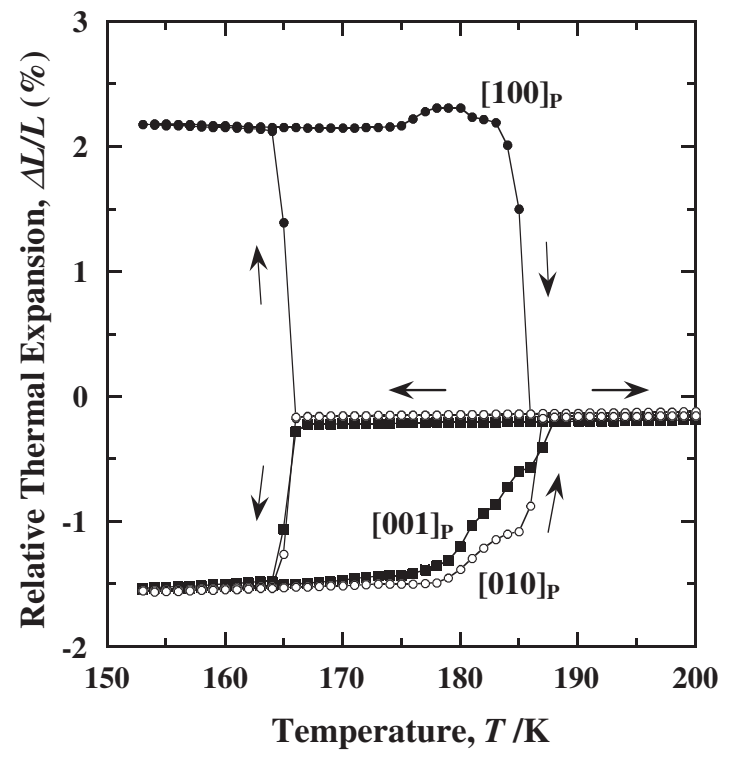

Fig. 2 Spontaneous linear thermal expansion curves of the single crystal $\mathrm{Fe}_{22.0} \mathrm{Ni}_{51.5} \mathrm{Ga}_{26.5}$ alloy. The arrow directions indicate the heating and cooling processes.

as $T_{\mathrm{C}}=370 \mathrm{~K}, M_{\mathrm{s}}=170 \mathrm{~K}, M_{\mathrm{f}}=168 \mathrm{~K}, A_{\mathrm{s}}=175 \mathrm{~K}$, and $A_{\mathrm{f}}=188 \mathrm{~K}$.

Figure 2 shows the spontaneous linear thermal expansion (LTE) curves of the $\mathrm{Fe}_{22.0} \mathrm{Ni}_{51.5} \mathrm{Ga}_{26.5}$ alloy. The arrows indicate the cooling and heating processes. The relative linear length change $(\Delta L / L)$ associated with the martensitic transformation in the $[100]_{\mathrm{P}}$ direction is about $+2.2 \%$, and those in the directions of $[010]_{\mathrm{P}}$ and $[001]_{\mathrm{P}}$ are about $-1.6 \%$ as shown in Fig. 2. Since the LTE measurement for each direction for $[100]_{\mathrm{P}},[010]_{\mathrm{P}}$, and $[001]_{\mathrm{P}}$ in the parent cubic phase was carried out independently, the value of the LTE and the temperature $A_{\mathrm{f}}$ exhibit slightly different value in each measurement, because the appearance and disappearance of the martensite phase in a self-accommodating manner are different, depending on defects and internal stresses. Therefore, the volume change associated with the martensitic transformation is not in accord with the data obtained from the change in the lattice constant. The transformation temperature interval between $M_{\mathrm{s}}$ and $M_{\mathrm{f}}$ is $2 \mathrm{~K}$, and the LTE sharply changes with the martensitic transformation in the cooling process. On the other hand, the interval between $A_{\mathrm{s}}$ and $A_{\mathrm{f}}$ is $13 \mathrm{~K}$, and the LTE gradually changes with the reverse transformation in the heating process. These behaviors of the LTE are in accord with these of the thermomagnetization curves. These results are caused by the difference in the processes between disappearance and appearance of the martensite phase. Recently, Oikawa et al. ${ }^{12)}$ have reported that an $\mathrm{Fe}_{19.0} \mathrm{Ni}_{54.0} \mathrm{Ga}_{27.0}$ alloy quenched from $1473 \mathrm{~K}$ martensitically transforms from bcc to a $14 \mathrm{M}$ structure with a 7-layer modulation. The lattice parameters in the martensite phase for the reported alloy are $a_{14 \mathrm{M}}=0.427 \mathrm{~nm}, \quad b_{14 \mathrm{M}}=0.270 \mathrm{~nm}, \quad c_{14 \mathrm{M}}=2.930 \mathrm{~nm}$, $\beta_{14 \mathrm{M}}=86.6^{\circ}$, and $\mathrm{L} 2{ }_{1}$-Heusler structure in the parent phase is $a_{\mathrm{L} 2_{1}}=0.576 \mathrm{~nm}$. Assuming that the crystal structures in the present study are the same as those of the reported alloy, the directions $\langle 100\rangle_{\mathrm{P}}$ in the parent cubic phase correspond to the direction $[701]_{14 \mathrm{M}},[70 \overline{1}]_{14 \mathrm{M}}$ or $[010]_{14 \mathrm{M}}$ in the $14 \mathrm{M}$ 
martensite phase. By considering the change in the lattice constants, the $[010]_{14 \mathrm{M}}$ is decreased but the $[701]_{14 \mathrm{M}}$ and $[70 \overline{1}]_{14 \mathrm{M}}$ are increased through the martensitic transformation. As shown in Fig. 2, only the direction $[100]_{\mathrm{P}}$ is increased and other directions are decreased through the martensitic transformation. Therefore, the $[010]_{14 \mathrm{M}}$ direction of the martensite phase is preferentially developed in the parent directions $[010]_{\mathrm{P}}$ and $[001]_{\mathrm{P}}$.

The magnetization curves in the zero-field cooling (ZFC) state for the single crystal $\mathrm{Fe}_{22.0} \mathrm{Ni}_{51.5} \mathrm{Ga}_{26.5}$ alloy of the $\beta^{\prime}$ martensite phase are shown Fig. 3. The magnetizations in the parent directions $[100]_{\mathrm{P}},[010]_{\mathrm{P}}$ and $[001]_{\mathrm{P}}$ were measured in the maretensite state at $T=10 \mathrm{~K}$. The measured $M-H$ curves for the directions $[010]_{\mathrm{P}}$ and $[001]_{\mathrm{P}}$ are situated above the curve for the direction $[100]_{\mathrm{P}}$. It should be noted that the twin boundary motion by applying magnetic field is easiest around $M_{\mathrm{f}}$, and therefore the change of the variants at $10 \mathrm{~K}$ far below the $M_{\mathrm{f}}$ would be difficult. Taking the LTE curves given in Fig. 2 into account, the easy axis is considered to be located near the normal directions to the $[010]_{14 \mathrm{M}}$ corresponding to the directions $[010]_{\mathrm{P}}$ and $[001]_{\mathrm{P}}$. The present data imply that the $\beta^{\prime}$ martensite phase is incomplete in the single-variant state, that is, some variants would exist in the $\beta^{\prime}$ martensite phase as a multi-variant state because the LTE change in the curve due to the transformation is lower than the value expected from the lattice constants. Since the magnetocrystalline anisotropy is dispersed in the multi-variant state, the magnetization process in the multi-variant state does not agree with that in the single variant-state, even though the saturation field in the multi-variant state coincides with that in the single-variant state. In the present data, therefore, the magnetic field at which the curve of the measurement along the direction $[100]_{\mathrm{P}}$ rides on the curve along the direction $[001]_{\mathrm{P}}$ is defined as the magnetic anisotropy field $H_{\mathrm{A}}$. Strictly speaking, after correcting the demagnetizing field, the value of the magnetocrystalline anisotropy constant was evaluated from the saturation magnetization $M_{\text {sat }}$ and the magnetic

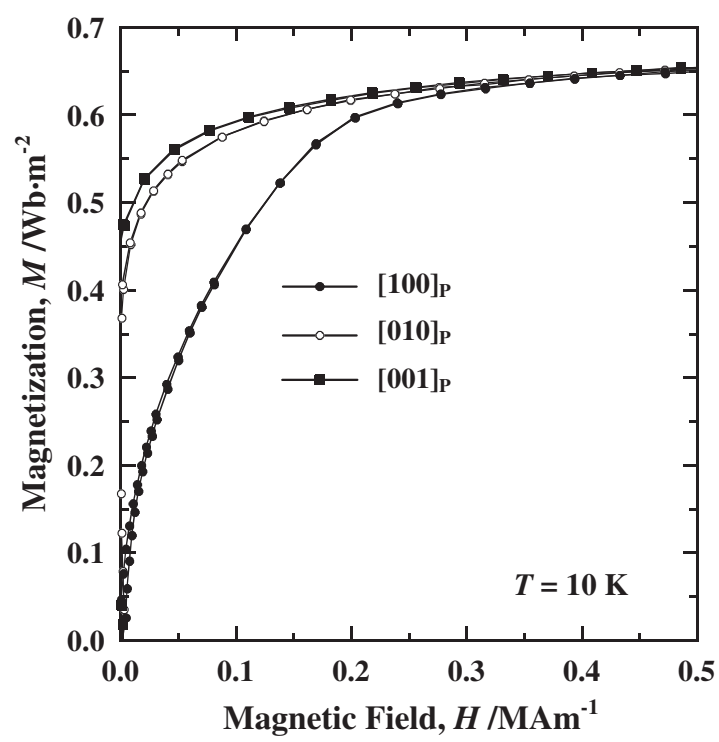

Fig. 3 Magnetization curves along the parent directions $[100]_{\mathrm{P}},[010]_{\mathrm{P}}$ and $[001]_{\mathrm{P}}$ for the single crystal $\mathrm{Fe}_{22.0} \mathrm{Ni}_{51.5} \mathrm{Ga}_{26.5}$ alloy in the $\beta^{\prime}$ martensitic phase. anisotropy field $H_{\mathrm{A}}$. Accordingly, the magnetocrystalline anisotropy constant $K$ for the $\mathrm{Fe}_{22.0} \mathrm{Ni}_{51.5} \mathrm{Ga}_{26.5}$ alloy in the $\beta^{\prime}$ martensite phase is estimated to be about $1.3 \times 10^{5}\left(\mathrm{~J} / \mathrm{m}^{3}\right)$ from the present data by using the following expression.

$$
K=\frac{M_{\mathrm{sat}} \cdot H_{\mathrm{A}}}{2}
$$

In comparison with the isotropic parent cubic phase, the $14 \mathrm{M}$ martensite phase exhibits a strong magnetocrystalline anisotropy, being the same order of that of $\mathrm{CoNiAl}^{9)}$ and $\mathrm{Ni}_{2} \mathrm{MnGa}^{15}$ ) The MFIS depends on the magnetocrystalline anisotropy and the mobility of twin boundaries as mentioned in introduction. To move the twin boundaries, it is required that the magnetocrystalline anisotropy energy is larger than the driving energy to move the twin boundaries. The Fe-NiGa alloy system is expected to have low twinnig stresses, because this system undergoes a thermoelastic martensitic transformation from a B2 and/or an L2 1 -Heusler parent to a martensite phase, following by a 7-layer modulated (14 M) structure together with a 5-layer modulated (10 M) structure ${ }^{13)}$ in analogy with $\mathrm{Ni}_{2} \mathrm{MnGa}{ }^{14)}$ Accordingly, a large MFIS is expected in the present alloy system. Details of these results will be reported in the near future.

\section{Conclusion}

The magnetic easy axis and the magnetocrystalline anisotropy in a single crystal $\mathrm{Fe}_{22.0} \mathrm{Ni}_{51.5} \mathrm{Ga}_{26.5}$ ferromagnetic shape memory alloy (FSMA) have been investigated. The data on the spontaneous $(H=0)$ linear thermal expansions (LTE) and the magnetization curve for the $\beta^{\prime}$ martensite phase tell us that the magnetic easy axis is the $[010]_{14 \mathrm{M}}$. The magnetocrystalline anisotropy constant $K$ related to the magnitude of the magnetic-field-induced strains (MFISs) in the single crystal $\beta^{\prime}$ martensite phase is large and estimated to be about $1.3 \times 10^{5} \mathrm{~J} / \mathrm{m}^{3}$. From the present results, it is expected that the $\mathrm{Fe}-\mathrm{Ni}-\mathrm{Ga}$ ferromagnetic shape memory alloy system exhibits a large value of the MFIS.

\section{Acknowledgement}

A part of the present study was supported by the Grant-inaids for Scientific Research from the Ministry of Education, Culture, Sports, Science and Technology, Japan.

\section{REFERENCES}

1) K. Ullakko, J. K. Huang, C. Kantner, R. C. O'Handley and V. V. Kokorin: Appl. Phys. Lett. 69 (1996) 1966-1968.

2) R. D. James and M. Wuttig: Philos. Mag. A77 (1998) 1273-1299.

3) T. Kakeshita, T. Takeuchi, T. Fukuda, T. Saburi, R. Oshima and S. Muto: Appl. Phys. Lett. 77 (2000) 1502-1504.

4) F. Gejima, Y. Sutou, R. Kainuma and K. Ishida: Metall. Mater. Trans. A 30 (1999) 2721-2723.

5) A. Fujita, K. Fukamichi, F. Gejima, R. Kainuma and K. Ishida: Appl. Phys. Lett. 77 (2000) 3054-3056.

6) M. Wuttig, J. Li and C. Craciunescu: Scr. Mater. 44 (2001) 2393-2397.

7) K. Oikawa, T. Ota, F. Gejima, T. Ohmori, R. Kainuma and K. Ishida: Mater. Trans. 42 (2001) 2472-2475.

8) K. Oikawa, L. Wulff, T. Iijima, F. Gejima, T. Ohmori, A. Fujita, K. Fukamichi, R. Kainuma and K. Ishida: Appl. Phys. Lett. 79 (2001) 3290-3292. 
9) H. Morito, A. Fujita, K. Fukamichi, R. Kainuma, K. Ishida and K. Oikawa: Appl. Phys. Lett. 81 (2002) 1657-1659.

10) Y. Murakami, D. Shindo, K. Oikawa, R. Kainuma and K. Ishida: Acta Mater. 50 (2002) 2173-2184.

11) R. C. O'Handley: J. Appl. Phys. 83 (1998) 3263-3270.

12) K. Oikawa, T. Ota, Y. Sutou, T. Ohmori, R. Kainuma and K. Ishida: Mater. Trans. 43 (2002) 2360-2362.
13) K. Oikawa, T. Ota, T. Ohmori, Y. Tanaka, H. Morito, A. Fujita, R. Kainuma, K. Fukamichi and K. Ishida: Appl. Phys. Lett., 81 (2002) 5201-5203.

14) A. Sozinov, A. A. Likhachev and K. Ullakko: IEEE Trans. Magn. 38 (2002) 2814-2816.

15) R. Tickle and R. D. James: J. Magn. Magn. Mater. 195 (1999) 627-638. 